\title{
The Cell versus the System: Standardization challenges for electricity storage devices
}

\author{
P. Van den Bossche ${ }^{1}$, F. Van Mulders ${ }^{1}$, B. Verbrugge ${ }^{1}$, N. Omar ${ }^{2}$, H. Culcu ${ }^{2}$, J. Van Mierlo ${ }^{2}$ \\ ${ }^{1}$ Erasmus University College Brussels, IWT \\ Nijverheidskaai 170,1070 Anderlecht, Belgium peter.van.den.bossche@docent.ehb.be \\ ${ }^{2}$ Vrije Universiteit Brussel, Pleinlaan 2, 1050 Elsene, Belgium
}

\begin{abstract}
The introduction of new electricity storage components such as electric double-layer capacitors or lithium batteries creates the necessity for relevant and reliable standards to assess components' performance and safety. Technical committees within international standardization bodies such as IEC and ISO have thus increased the intensity of standardization work on the matter. The paper will describe current work on the matter, concentrating on the characteristic action points where standardization work is needed.
\end{abstract}

Keywords: standardization, battery, EDLC, RCS,secondary battery

\section{Introduction}

In urban traffic, due to their beneficial effect on environment, electrically propelled vehicles are an important factor for improvement of traffic and more particularly for a healthier living environment. The operation of the electrically propelled vehicle is dependent on the availability of efficient electric energy storage devices. New types of storage devices such as electric double-layer capacitors (commonly known as supercapacitors) and high-capacity batteries such as lithium batteries (which can be designed for either high energy or high power applications) are now appearing on the market and promise to make a significant contribution to de deployment of performant electrically propelled vehicles, both battery-electric and hybrid.

The comparison between energy storage devices is made obvious in the so-called Ragone chart 1 , where one can see the balance between specific energy and specific power. The areas corresponding to each electrochemical couple show different implementations of this technology for specific applications.

The introduction of such new components creates the necessity for relevant and reliable standards to assess components' performance and safety. Technical committees within interna- tional standardization bodies such as IEC and ISO have thus increased the intensity of standardization work on the matter.

\section{The standardization of electric double-layer capacitors}

Work is now being performed by IEC TC69 on defining a standard procedure to measure electrical characteristics of the electric double-layer capacitors. This work will lead to the international standard IEC 62576 "Electric double-layer capacitors for use in hybrid electric vehicles - Test methods for electrical characteristics". [1]

The main parameter of the capacitor, its capacitance, is obtained by the so-called energy conversion capacitance method. The stored energy in a capacitor charged to a voltage $U$ is being given by

$$
W=\frac{C \cdot U^{2}}{2}
$$

so the capacitance when the capacitor is discharged with a constant current between two set voltage levels, can be derived from energy dis- 


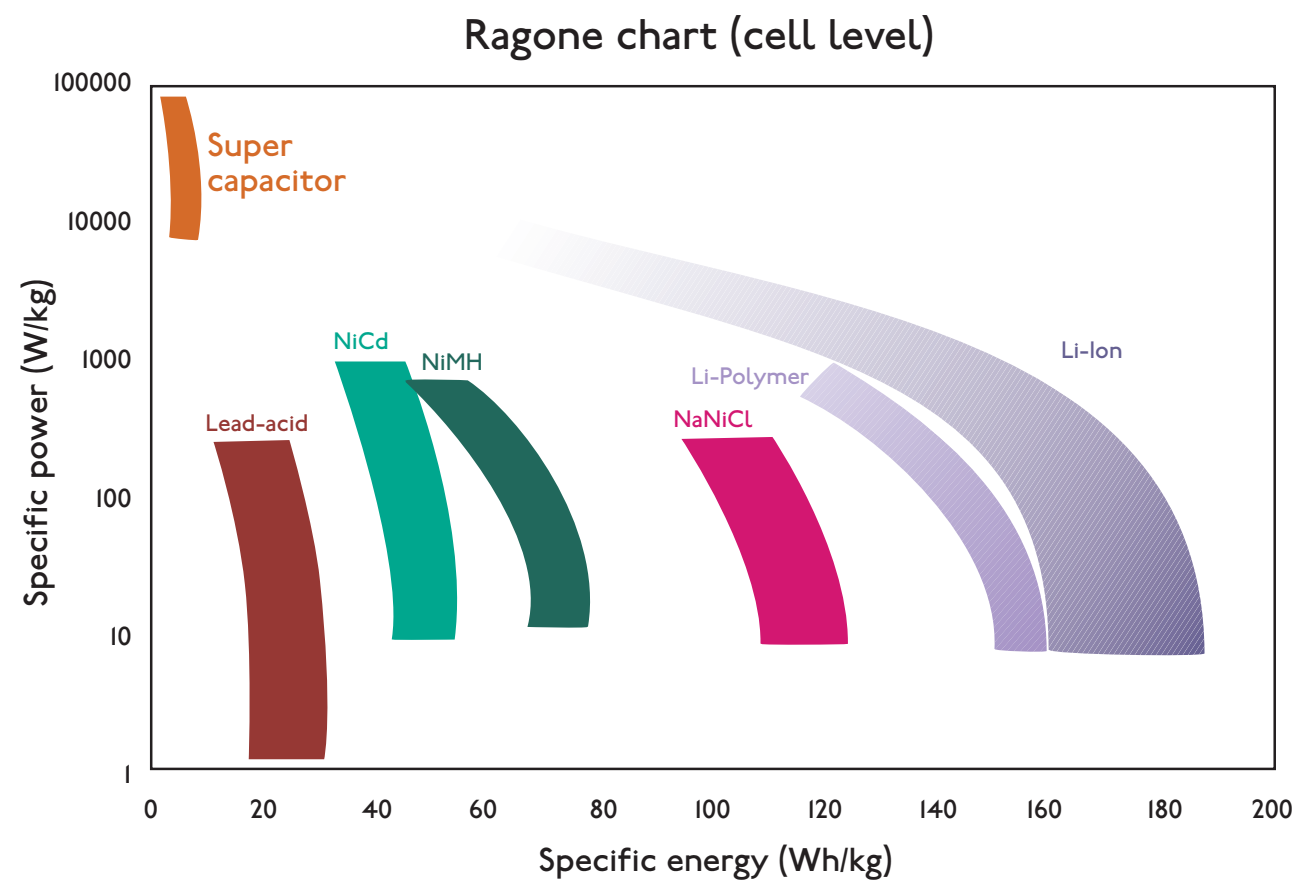

Figure 1: Ragone diagram

charged according to

$$
C=\frac{2 W}{\left(0,9 U_{R}\right)^{2}-\left(0,7 U_{R}\right)^{2}}
$$

A further key parameter for power transfer is the internal resistance. This can be derived from the initial voltage drop at the start of discharge 2 and is given by

$$
R=\frac{\Delta U_{3}}{I_{d}}
$$

This will allow to derive the maximum power

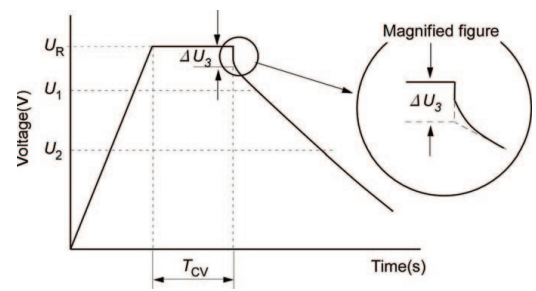

Figure 2: Measurement of internal resistance

density, for which the ?matched impedance? method is chosen: the power delivered will be maximal when the resistance of the load equals the internal resistance; in this case, the voltage over the load equals the voltage drop over the internal resistance and the power is given by

$$
P_{d m}=\frac{\frac{U_{R}^{2}}{2}}{R_{i} \cdot m}=\frac{U_{R}^{2}}{4 \cdot R_{i} \cdot m}
$$

Furthermore, the voltage maintenance (self discharge) over a 72 hour period is measured. The energy efficiency of the capacitor are calculated as the ratio of charged and discharged energy between half and full rated voltage, as this is the area where the capacitor is most likely to be used:

$$
\eta=\frac{E_{d}}{E_{c}}=\frac{\int_{t_{U_{R}}}^{\frac{t_{\frac{U}{R}}}{2}} I_{d} \cdot U(t) d t}{\int_{t_{\frac{U_{R}}{2}}^{t_{U_{R}}}}^{t_{2}} \cdot U(t) d t}
$$

Work on this standard is well advancing and it is now being circulated as CDV, aiming for publication in 2010 .

\section{The standardization of lithium secondary batteries for auto- motive traction}

The standardization scene for automotive lithium batteries presents a more complicated challenge. 
On one hand one has to take into account the desired application. The charge and discharge characteristics of batteries in hybrid applications, where the battery is intended foremostly as power storage, differ strongly from batteryelectric applications, where the battery acts as energy storage, so specific test cycles have to be defined. Furthermore, battery characteristics, particularly concerning safety, can be regarded from the viewpoint of the battery or from the viewpoint of the system. The first approach is the traditional battery cell and module standardization as performed by the IEC battery committees. The automotive sector however, represented in the relevant ISO committees, wants to standardize the battery system, as a component of the vehicle traction system. As has happened in the past [2] on other electric vehicle related issues, the different standardization cultures between the two sectors have come to a collision, with several overlapping and potential conflicting new work items on lithium batteries being submitted in parallel. High level negotiations have been held to resolve these problems, leading to the definition of clear scopes for new standards to be drafted on the subject in order to avoid double work. A consensus agreement will allow the following work items to proceed:

- ISO TC22 SC21 will focus on the system approach, i.e. the battery integrated into the vehicle system.

- A joint working group from IEC TC21/SC21A/TC69 will draft standards for battery cells and modules for automotive use, closely complementing the ISO document through committee liaisons. The boundary between the IEC and ISO documents will be drawn based on the definition of the battery pack and its associated ancillaries which are part of the vehicle battery system but outside the battery as such.

- General, non-automotive, lithium battery standards will be treated by IEC SC21A.

\section{ISO TC22 SC21 work on lithium batteries}

Work is now ongoing for the future ISO 12405 "Electrically propelled road vehicles - Test specification for lithium-ion traction battery packs and systems" for which there will be two parts, taking into account the specific needs of high power and high energy systems. [3] This document reflects the standardization needs of vehicle manufacturers, and is a synthesis of test procedures developed by EUCAR, FreedomCar and USABC. It describes performance, reliability and abuse procedures for the battery system 3 .

The first part, for high power applications, focuses on determining pulse power capability of the battery, with specific cycles to measure the momentary voltage drop during discharge or charge microcycles 4 , which allows to assess the internal resistance and maximum power for several burst times, and cycle tests mimicking the sequencing of short discharge and charge microcycles with high current bursts as are typically found in the operation of a battery in a hybrid vehicle 5. It is clear that this mode of operation creates the need for specific test cycles different from the standard capacity tests developed for battery-electric vehicles.

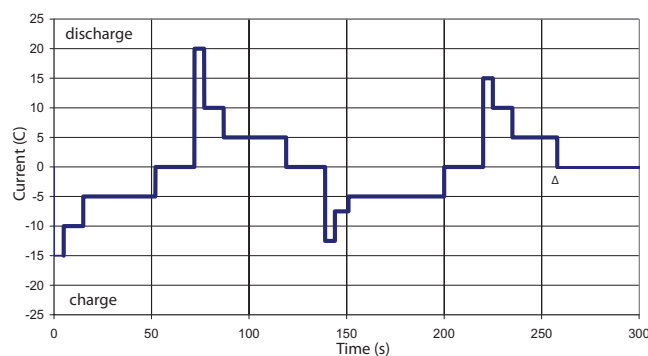

Figure 4: Momentary voltage drop for internal resistance

Since the ISO 12405 document is still under development, these cycles are given only as examples to highlight there characteristics and should be not considered as definitive specifications. This applies of course to the new IEC standards discussed below too.

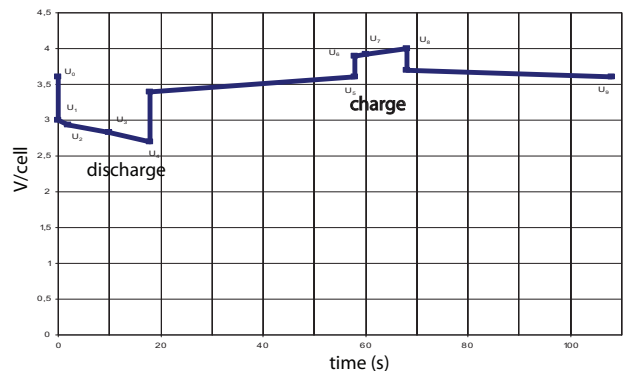

Figure 5: ISO Micro-cycle for hybrid vehicle

Since the ISO 12405 document is still under development, these cycles are given only as examples to highlight there characteristics and should be not considered as definitive specifications. This applies of course to the new IEC standards discussed below too.

\section{IEC TC21/SC21A/TC69 IEC TC21/SC21A/TC69}

As a battery system is always consisting of individual cells or batteries, specific standards have 


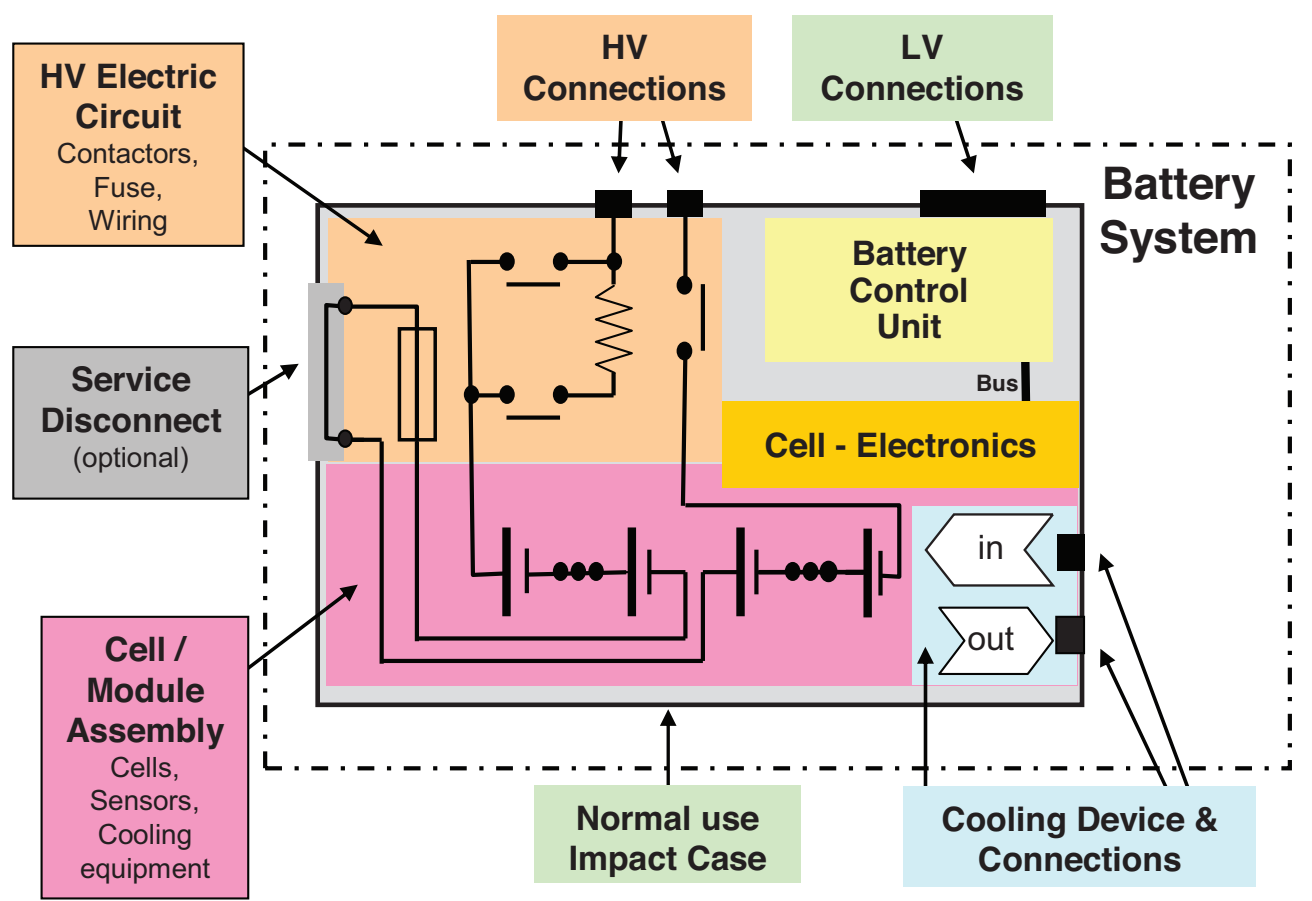

Figure 3: Battery system on a vehicle

also to be drafted on cell level. This standardization always has been the province of IEC, which now will complement the ISO 12405 document with two new standards being drafted as parts of IEC 61982 "Secondary batteries for the propulsion of electric road vehicles". The first three parts of this standard, already published in the first years of this decennium, provide generic performance tests of traction batteries.

Part 4 [4] will specify performance tests for lithium ion cells and batteries, which is deemed necessary for securing a basic level of performance and obtaining essential data for vehicle and system design.

The standard, now circulating as a committee draft, provides capacity, power density and energy density tests, where current levels and cycle are chosen specifically in function of the desired application (battery electric or hybrid electric vehicle). The same division applies to the life cycle testing, where a typical microcycle for battery electrics 6 contrasts with a microcycle for hybrids 7.

Although the battery electric microcycle contains a charging phase which corresponds to regenerative braking, its overall characteristic is chargedepleting, and microcycles are followed in sequence until the battery is exhausted to produce a full discharge cycle. The hybrid microcycle however is characterised by charge and discharge bursts with an overall charge balance of near zero (actually slightly positive to take into account charge efficiency), corresponding to the battery use in a non-depleting hybrid.

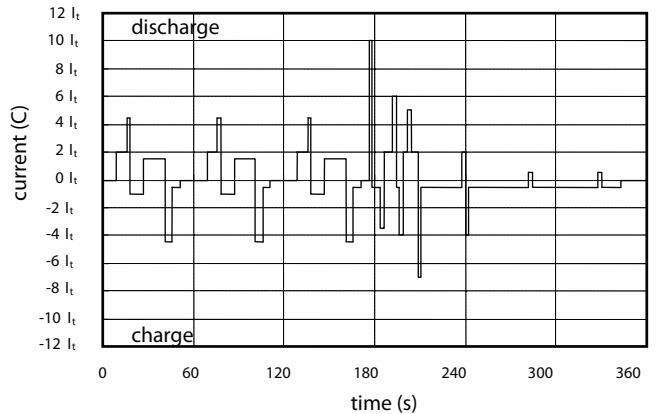

Figure 6: IEC Micro-cycle for battery-electric

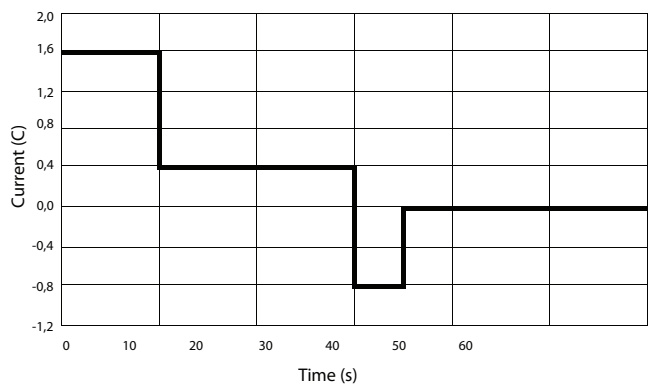

Figure 7: IEC Micro-cycle for hybrid 
Part 5 [5] of IEC 61982 will deal with safety, providing a set of tests to assess cell behaviour when subject to abuse conditions, both mechanical (vibration, shock and crush), thermal and electrical (short circuit and overcharge), with tests results in ascending order of severity going from no effect to explosion of the cell. The main difference with the safety and abuse tests in ISO 12405 is that it is the cell itself which is subject to abuse directly and that safety functions of the battery system which may cut off a circuit for example are not considered.

\section{Future challenges with new components}

The publication of the aforementioned international standards will provide valuable guidelines for component and system developers as well as allowing performance evaluation for systems using electric double-layer capacitors or performance batteries. However, new components are being developed such as the "lithium capacitor" [6], a device having characteristics of both a battery and a capacitor, and for which the existing measurement procedures for each of these devices may not be appropriate. The deployment of such devices in the market may necessitate the drafting of specific standards.

\section{Conclusions}

The study of the standardization process for electricity storage devices allows to draw interesting insights in the general impact of standards, proving on one hand how international standardization does provide a direct benefit to technological and societal development through the deployment of electrically propelled vehicles, and highlighting on the other hand the dynamics of the international standardization world. The proposed work for developing lithium ion battery standards saw a resurgence of the eternal conflict between ISO and IEC on electric vehicle standardization [2], which has deeper roots than just defining the right committee to do the job, as the differences in standardization culture and attitude between automotive manufacturers on one hand and the electrotechnical industry on the other hand often has impeded proper collaboration between committees and following different approaches, which when not resolved, will result in double work and the drafting of conflicting and eventually useless standards. These differences have been resolved in a climate of collaboration and consensus where all stakeholders agree to work together towards a common objective highlighting the benefits of international standardization for the common good.

\section{References}

[1] IEC 62576/CDV, Electric Double-Layer Capacitors for Use in Hybrid Electric Vehicles - Test Methods for Electrical Characteristics, 2008.

[2] P. Van den Bossche, The electric vehicle, raising the standards, $\mathrm{PhD}$ thesis, Vrije Universiteit Brussel, 2003.

[3] ISO 12405/CD, Electrically propelled road vehicles - Test specification for lithium-ion traction battery packs and systems - Part 1: High power applications, 2009.

[4] IEC 61982-4/NP, Electrically propelled road vehicles - Test specification for lithium-ion traction battery packs and systems - Part 1: High power applications, 2008.

[5] IEC 61982-5/NP, Secondary batteries for the propulsion of electric road vehicles - Safety testing for lithium-ion cells and batteries, 2008.

[6] G. Sikha et.al., Mathematical model for a lithium-ion battery/electrochemical capacitor hybrid system, Journal of the Electrochemical Society, 152(8), A1682-1693

\section{Authors}

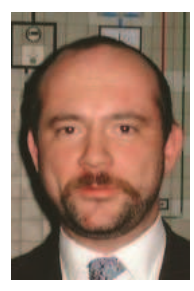

Peter Van den Bossche promoted in Engineering Sciences from the Vrije Universiteit Brussel on a thesis "The Electric vehicle, raising the standards". $\mathrm{He}$ is currently lecturer at the Erasmushogeschool Brussel and the Vrije Universiteit Brussel. Since more than 15 years he is active in several international standardization committees, currently acting as Secretary of IEC TC69.

Frederik Van Mulders graduated in 2005 as a Mechanical Industrial Engineer at the Erasmus University College Brussels and was invited to be a $\mathrm{PhD}$ student at the ETECdepartment for the Vrije Universiteit Brussel and the Erasmus University College Brussels. There, his main research covers supercapacitor based peak power units. 

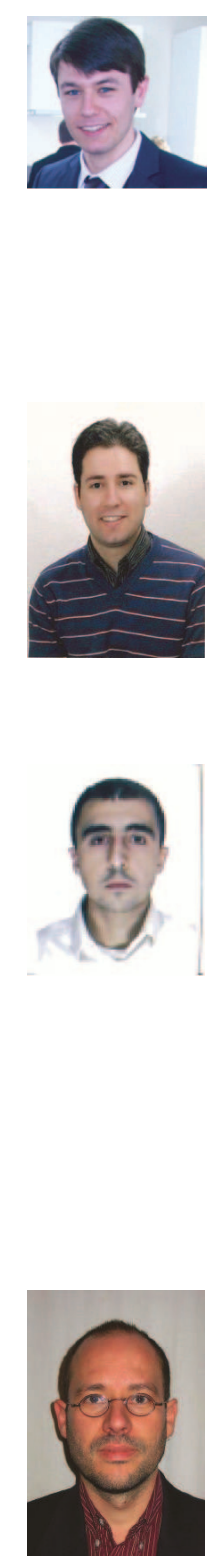

Bavo Verbrugge graduated as a Master in industrial engineering in 2007. At this time he works as a $\mathrm{PhD}$. candidate at the free University of Brussels (VUB), where he's currently performing research on integrated modelling of batteries and EDLC's.

Noshin Omar was born in Kurdistan, in 1982. He obtained the M.S. degree in Electronics and Mechanics from Hogeschool Erasmus in Brussels. He is currently pursuing the PHD degree in the department of Electrical Engineering and Energy Technology ETEC, at the Vrije Universiteit Brussel, Belgium. His research interests include applications of supercapacitors and batteries in HEV?s.

Hasan CULCU (born in Isparta, Turkey, 1982) obtained his B.S. and M.S. degree in Mechanical and Electrical Engineering at Vrije Universiteit Brussel, where he is doing now his research concerning energy storage systems and simulations of hybrid vehicles.

Joeri van Mierlo received M.S. and $\mathrm{PhD}$ degree in electromechanical engineering from Vrije Universiteit Brussel in 1992 and 2000 with greatest distinction. From 2004 he has been appointed as a fulltime professor at the Vrije Universiteit Brussel. Currently his research is devoted to the development of hybrid propulsion (converters, supercaps, energy management, etc.) systems as well as to the environmental comparison of vehicles with different kind of drive trains and fuels. 